\title{
MONOTONICITY OF THE FORCING TERM AND EXISTENCE OF POSITIVE SOLUTIONS FOR A CLASS OF SEMILINEAR ELLIPTIC PROBLEMS
}

\author{
GADAM SUDHASREE
}

(Communicated by Barbara L. Keyfitz)

\begin{abstract}
We study the existence of positive solutions to the equation $\Delta u+$ $f(u)+\lambda g(\|x\|)=0$ in the unit ball in $\mathbb{R}^{N}$ with Dirichlet boundary conditions, where $f$ is superlinear with $f(0)=0$ and $\lambda$ is a real parameter. We prove that if $g$ is monotonically increasing, then there exists an $\alpha<0$ such that for $\lambda<\alpha$ the above equation has no positive solution. This is in contrast to the case of $g$ monotonically decreasing, where positive solutions exist for all negative values of $\lambda$.
\end{abstract}

\section{INTRODUCTION}

Let $g:[0,1] \rightarrow(0, \infty)$ be a continuous, nondecreasing function and $\lambda \in \mathbb{R}$. Here we consider the existence of positive solutions to the equation

$$
\begin{aligned}
\Delta u+f(u)+\lambda g(\|x\|) & =0 & & \text { in } B_{1}, \\
u & =0 & & \text { on } \partial B_{1},
\end{aligned}
$$

as $\lambda$ varies over $\mathbb{R}$, where $B_{1}$ denotes the open unit ball in $\mathbb{R}^{N}$ centered at the origin and $f:[0, \infty) \rightarrow[0, \infty)$ is differentiable, satisfying

$$
f(0)=0, \quad f^{\prime \prime}(t) \geq 0 \quad \text { with } \lim _{t \rightarrow \infty}(f(t) / t)=\infty .
$$

We prove that for $\lambda$ 's with $|\lambda|$ large (1.1) does not have positive solutions. This contrasts with the case in which $g$ is monotonically decreasing [2]. Indeed, taking $f(t)=t^{p}$, with $1<p<(N+2) /(N-2), u_{0}$ the positive radial solution of

$$
\begin{aligned}
\Delta w+w^{p}=0 & \text { in } B_{1}, \\
w=0 & \text { on } \partial B_{1},
\end{aligned}
$$

and then taking $g(r)=u_{0}^{p}(r)$, it is easily seen that (1.1) has a positive solution for each $\lambda \leq 0$.

Our results extend and complement the work of Ramaswamy [4] and of Brown, Castro, and Shivaji [1].

Received by the editors April 9, 1990 and, in revised form, May 29, 1990.

1980 Mathematics Subject Classification (1985 Revision). Primary 35J25; Secondary 35P99. 
Theorem 1. If (1.2) holds then there exist $\alpha<0$ and $\beta>0$ such that, for $\lambda \notin[\alpha, \beta]$, equation (1.1) has no positive solutions.

Proof. The existence of $\beta$ follows from a simple integration-by-parts argument using the fact that $f$ is superlinear.

Let $\lambda$ be negative. From Gidas, Ni, and Nirenberg [3] it follows that any positive solution to (1.1) is radially symmetric and decreasing. Hence (1.1) is equivalent to the singular ordinary differential equation

$$
\begin{gathered}
u^{\prime \prime}+\frac{n}{r} u^{\prime}+f(u)-\lambda g(r)=0 \quad \text { in }(0,1), \\
u^{\prime}(0)=0, \quad u(1)=0,
\end{gathered}
$$

where $\lambda>0$ and $n=N-1$. If we assume, on the contrary, that such an $\alpha$ does not exist, then there exists a sequence $\left(u_{j}, \lambda_{j}\right)$ satisfying (1.3) with $\lambda_{j} \rightarrow \infty$. Since $u=u_{j}$ is radially decreasing on $[0,1]$, we have $\Delta u \leq 0$ at 0 and $\Delta u>0$ at 1 . Thus there exists $a_{j} \in[0,1)$ such that $\Delta u=0$ at $a_{j}$. That is,

$$
f\left(u\left(a_{j}\right)\right)=\lambda_{j} g\left(a_{j}\right)
$$

Next we prove that $a_{j} \rightarrow 1$ as $\lambda_{j} \rightarrow \infty$. Since $u$ is radially decreasing, there exists a $b_{j} \in\left(a_{j}, 1\right)$ such that

$$
2 f\left(u\left(b_{j}\right)\right)=\lambda_{j} g\left(a_{j}\right) .
$$

Since $g$ is nondecreasing, we have $a_{j}<b_{j}$. This and (1.5) imply that

$$
\lambda_{j} g(0) \leq 2 f\left(u\left(b_{j}\right)\right)=\lambda_{j} g\left(a_{j}\right) \leq \lambda_{j} g\left(b_{j}\right) \leq \lambda_{j} g(1) .
$$

Because of (1.6), for $t \in\left[b_{j}, 1\right]$, we have

$$
\left(t^{n} u^{\prime}\right)^{\prime}=t^{n}\left(\lambda_{j} g(t)-f(u(t))\right) \geq \frac{1}{2} t^{n} \lambda_{j} g(t),
$$

using the fact that $f, g$ are nondecreasing and $u$ is decreasing. (Note that (1.2) forces $f$ to be nondecreasing.) Now, integrating on $\left[b_{j}, 1\right]$, we get

$$
u^{\prime}(1)-b_{j}^{n} u^{\prime}\left(b_{j}\right) \geq \lambda_{j} g\left(b_{j}\right)\left(1-b_{j}^{N}\right) /(2 N) .
$$

Thus

$$
g(0) \lambda_{j}\left(1-b_{j}^{N}\right) \leq 2 N\left(b_{j}^{n}\left|u^{\prime}\left(b_{j}\right)\right|-\left|u^{\prime}(1)\right|\right),
$$

using (1.6) and, since $u^{\prime}\left(b_{j}\right)<0, u^{\prime}(1) \leq 0$. (Also note that our choice of $a_{j}$ as in (1.4) implies that $u^{\prime \prime}>0$ in $\left(a_{j}, 1\right)$.) Now, multiplying (1.3) by $r^{2 n} u^{\prime}$ and integrating on $\left[b_{j}, 1\right]$ we obtain

$$
\begin{aligned}
\left(u^{\prime}(1)\right)^{2}-b_{j}^{2 n}\left(u^{\prime}\left(b_{j}\right)\right)^{2} & \geq 2 b_{j}^{2 n} F\left(u\left(b_{j}\right)\right)+4 n \int_{b_{j}}^{1} r^{2 n-1} F(u)+2 \lambda_{j} \int_{b_{j}}^{1} r^{2 n} g u^{\prime} \\
& >2 \lambda_{j} \int_{b_{j}}^{1} r^{2 n} g u^{\prime} \geq-2 \lambda_{j} g(1) u\left(b_{j}\right) .
\end{aligned}
$$


This and (1.5) yield

$$
b_{j}^{2 n}\left(u^{\prime}\left(b_{j}\right)\right)^{2}-\left(u^{\prime}(1)\right)^{2}<c f\left(u\left(b_{j}\right)\right) u\left(b_{j}\right),
$$

where $c$ is a constant independent of $j$. From (1.7) and (1.8), we have

$$
\left(1-b_{j}^{N}\right)^{2}<c_{1} u\left(b_{j}\right) / f\left(u\left(b_{j}\right)\right) \rightarrow 0
$$

as $j \rightarrow \infty($ see $(1.4)$ and $(1.5))$. Hence $b_{j} \rightarrow 1$ as $j \rightarrow \infty$.

Now, by the convexity of $f \circ u$ in $\left[a_{j}, 1\right]$, we infer that $f\left(u\left(\left(1+a_{j}\right) / 2\right)\right) \leq$ $f\left(u\left(a_{j}\right)\right) / 2=f\left(u\left(b_{j}\right)\right)$. Thus $\left(1+a_{j}\right) \geq 2 b_{j}$, proving that $a_{j} \rightarrow 1$ as $j \rightarrow \infty$.

Now let $\varepsilon>0$, and choose $j$ large enough such that $1-a_{j}<\varepsilon$. Let $\mu_{1}=\mu_{1}\left(-\Delta, B_{1-\varepsilon}\right)$ denote the first eigenvalue of $-\Delta$ in $B_{1-\varepsilon}$, a ball of radius $(1-\varepsilon)$ around the origin. That is,

$$
\begin{aligned}
-\Delta \varphi_{1} & =\mu_{1} \varphi_{1} & & \text { in } B_{1-\varepsilon}, \\
\varphi_{1} & =0 & & \text { on } \partial B_{1-\varepsilon},
\end{aligned}
$$

where $\varphi_{1}$ is chosen to be positive in $B_{1-\varepsilon}$. We choose $j$ large enough so that $f^{\prime}\left(u\left(a_{j}\right)\right)>\mu_{1}$. This is possible by virtue of (1.2), along with (1.4) and (1.6). Then $v(x)=u(x)-u\left(a_{j}\right)$ satisfies

$$
\begin{aligned}
-\Delta v & >\mu_{1}\left(-\Delta, B_{a_{j}}\right) v & & \text { in } B_{a_{j}}, \\
v & =0 & & \text { on } \partial B_{a_{j}},
\end{aligned}
$$

with $v>0$ in $B_{a_{j}}$, which yields a contradiction if we consider the eigenvalue problem (1.9) in $B_{a_{j}}$. Hence the theorem is proven.

Remark 1. In contrast, when $g$ is decreasing, we might have the existence of positive solutions for all $\lambda<0$. For example, when $f(t)=t|t|^{p-1}, 1<p<$ $(N+2) /(N-2)$ for $N \geq 3$, and $g(x)=u_{0}^{p}(x)$ for any $\lambda<0$, there is a positive radial solution of (1.1) given by $c u_{0}$, where $c$ is such that $c-c^{p}=\lambda$. Thus the monotonicity properties of $g$ and the positivity of $g$ on the boundary $\partial B_{1}$ play an important role in the existence results.

Remark 2. It can be easily seen that there is no nonradial degeneracy all along the positive solution curve for negative $\lambda$ 's as opposed to symmetry breaking in the case when $g$ is decreasing [2].

\section{REFERENCES}

1. K. J. Brown, A. Castro, and R. Shivaji, Non-existence of radially symmetric non-negative solutions for a class of semi-positone problems, Differential Integral Equations 4 (1989), 541-546.

2. G. Sudhasree, Symmetry breaking for a class of semilinear elliptic problems and the bifurcation diagram for a 1-dimensional problem, Rend. Circ. Mat. Palermo (to appear). 
3. B. Gidas, W. M. Ni, and L. Nirenberg, Symmetry and related properties via the maximum principle, Comm. Math. Phys. 68 (1979), 209-243.

4. M. Ramaswamy, On the global set of solutions to a nonlinear ODE-theoretical and numerical description, J. Differential Equations 65 (1986), 1-48.

Department of Mathematics, University of North Texas, Denton, Texas 76203 